\title{
Patch testing in fixed drug eruptions-a 20-year review
}

\author{
Pedro Andrade, Ana Brinca and Margarida Gonçalo \\ Department of Dermatology and Venereology, Coimbra University Hospital, 3000-075 Coimbra, Portugal \\ doi:10.1111/j.1600-0536.2011.01946.x
}

\section{Summary}

\begin{abstract}
Background. The fixed drug eruption is a common adverse drug reaction. Clear identification of the culprit drug is not always possible in the clinical setting, and oral rechallenge may induce new lesions or severe reactions.

Objectives. The main purpose of this study was to evaluate the diagnostic value of patch testing in establishing an aetiological diagnosis in fixed drug eruptions.

Method. A retrospective analysis was conducted evaluating 52 patients $(17 \mathrm{M} / 35 \mathrm{~F}$, mean age $53 \pm 17$ years) with clinical diagnoses of fixed drug eruptions submitted to patch tests in a 20-year period in a Dermatology Department. Nonsteroidal anti-inflammatory drugs (NSAID) were clinically suspected in $90.4 \%$ of the cases, followed by antibiotics $(28.9 \%)$ and paracetamol $(15.4 \%)$.

Results. Patch tests on pigmented lesions were reactive in 21 patients $(40.4 \%), 20$ of those to NSAID (nimesulide, piroxicam and etoricoxib) and 1 to an antihistamine (cetirizine). All patch tests using other drugs were negative, even under conditions of high clinical suspicion. Oral rechallenge allowed confirmation of drug imputability in 5 of 31 test-negative cases. Cross reactivity was frequently observed between piroxicam and other oxicams, and between different antihistamines.

Conclusions. Patch testing was shown to be a simple and safe method to confirm drug imputabililty in fixed drug eruption, mainly when NSAID or multiple drugs are suspected. Persistent lack of reactivity to drug classes such as antibiotics and allopurinol represent an important limitation.
\end{abstract}

Key words: allopurinol; fixed drug eruption; NSAID; oral rechallenge; patch tests.

The fixed drug eruption (FDE) is an common adverse drug reaction $(1,2)$, characterized by the sudden onset of single or multiple round oedematous erythemato-violaceous plaques. These present with sharply demarcated borders and, often, central bullous detachments within 48-hr of drug intake, and typically regress with hyperpigmented, postinflammatory macules (2-4). In extreme cases of generalized FDE, it may be difficult to make a clinical and/or histopathological distinction between FDE and toxic epidermal necrolysis (TEN) (1-3), particularly the

Correspondence: Pedro Andrade, Serviço de Dermatologia e Venereologia, Hospitais da Universidade de Coimbra, EPE, Praceta Mota Pinto, 3000-075 Coimbra, Portugal. Tel: +351239400420; Fax: +351239400490. E-mail: pedro.andrade@portugalmail.com

Conflicts of interest: The authors have declared no conflicts.

Accepted for publication 4 May 2011 rare form of pure plaque TEN. Many cases arise after oral intake of non-steroidal anti-inflammatory drugs (NSAIDs) or antibiotics, but several other drugs can be related to this adverse reaction $(2-4)$. Clear identification of the culprit drug is not always easy in the clinical setting $(2,3)$, particularly when FDE occurs for the first time, in the elderly, or in the context of multiple medications. Typically, reexposure to the culprit drug induces an acute flare in less than 24-hr, expressed by recurrence of inflammatory signs on residual hyperpigmented patches (3). However, in drug rechallenge tests (the classic gold standard diagnostic procedure for $\operatorname{FDE}(2,4))$, new lesions may arise, and, in some cases, severe reactions may be triggered, with extensive involvement of the skin and mucosae, bullous detachments and deleterious systemic effects and symptoms $(2,3)$; therefore, topical provocation by patch testing has been used in FDE patients as a safe alternative to identify the culprit drug (2-7). Despite its limitations (2), 
patch tests are useful in a significant number of patients, allowing a precise aetiological diagnosis and minimizing the risk of severe adverse events after systemic drug reexposure. Therefore, they have been widely recommended as the initial diagnostic tool in $\operatorname{FDE}(2,3,8)$.

In this context, other than a general characterization of the population with a clinical diagnosis of FDE, the main objective of this study was the evaluation of the diagnostic value of patch testing in FDE in confirming drug imputability and the determination of cross-reactivity between related drugs.

\section{Patients and Methods}

The study consisted of a retrospective descriptive analysis of all patients with a clinical diagnosis of FDE who were subjected to patch testing for identification of the culprit drug in the period from 1990 to 2009 (20 years) in our department. All patients were characterized according to their age and sex, the suspected drug (according to intrinsic clinical and temporal criteria of the French pharmacovigilance system (9), concerning the specific substance and respective drug class), and reactivity of patch tests. Six drug classes were considered, as follows: NSAIDs, antibiotics, antihistamines, allopurinol, paracetamol, and other drugs. Patients who were tested for drugs under conditions of high clinical suspicion (when no more than two specific drugs were clinically suspected, on the basis of temporal relationships and, eventually, accidental re-exposure) were identified for comparative interpretation of patch test results.

Patch tests were performed in all patients at least 6 weeks after resolution of a preceding acute flare of FDE. Allergens were tested in Finn Chambers ${ }^{\circledR}$ on Scanpor ${ }^{\circledR}$ tape (Epitest Ltd Oy, Tuusula, Finland), simultaneously on non-lesional back skin and on residual pigmented lesions. On non-lesional skin, tests were performed with complete drug class series selected according to the clinical suspicion (NSAIDs and/or antibiotics) and the suspected drugs. On residual pigmented lesional skin, only suspected and related drugs were used, depending on the number and size of pigmented patches available for test application. In all cases, the allergen concentration in petrolatum ranged between $1 \%$ and $20 \%$. In cases presenting with few pigmented patches, suspected allergens were preferably tested with higher concentrations, as detailed in Table 1. Most allergens were chemicals supplied by the pharmaceutical industry, with chemical purity $>95 \%$, and were prepared in pet. in our hospital; more recently, they were supplied by Chemotechnique Diagnostics ${ }^{\circledR}$ (Vellinge, Sweden). In some cases, as pure allergens could not be obtained, suspected drugs, such as etoricoxib, celecoxib, and clobazam, were prepared by using the powder of commercial tablets in pet. (active drug between 5\% and $20 \%$ ) (Table 1). Allergens were applied on lesional skin under occlusion for 1 day and on non-lesional skin for 2 days. Readings were conducted on D2 and D3 on non-lesional skin, and on D1, D2 and D3 on lesional skin. Reactions were considered to be positive if infiltrated erythema or more intense local reactions were observed. Local pruritus or erythema lacking infiltration were considered to indicate non-reactiveness.

In cases of high clinical suspicion with negative results, patch tests on lesional skin were often repeated, following local tape stripping, with the suspected allergen at the same concentration and, in some cases, at higher concentrations (up to $20 \%$ ). Following persistent negative results, oral rechallenge tests were performed in some cases, under close surveillance, with small amounts of the suspected drugs $(25-100 \%$ of the drug dose in the commercial formulation). Recurrence of inflammatory signs in previous hyperpigmented lesions or the onset of new lesions within 24-hr following oral drug intake was considered to indicate a positive reaction.

\section{Results}

In the 20-year period, a total of 52 patients with a clinical diagnosis of FDE were patch tested in our department for identification of the culprit drug, representing $1 \%$ of all patients patch tested for any reason in the same period. Most of these patients were female $(\mathrm{n}=35$, $67.3 \%)$ and $>50$ years old $(\mathrm{n}=29,55.8 \%)$. The age distribution within the group was very homogeneous (Table 2), extending from 20 to 78 years, with a mean of $53 \pm 17$ years.

In half of the patients $(\mathrm{n}=26)$, drugs from a single drug class were clinically suspected of triggering FDE, whereas in the remaining 26 , multiple drug classes were considered. In the first group, NSAIDs were clearly the most suspected drugs (in 21 patients), followed by antibiotics $(n=2)$, paracetamol $(n=1)$, allopurinol $(n=1)$, and antihistamines $(\mathrm{n}=1)$. In the second group, NSAIDs were clinically suspected in all 26 patients, in association with antibiotics in 13 , with paracetamol in 7 , with allopurinol in 3, and with other drugs in 6, namely antihypertensive drugs, muscle relaxants, anticonvulsants, benzodiazepines, and other anti-inflammatory drugs.

This means that NSAIDs, in general terms, were the most commonly suspected drugs in the clinical setting in all FDE patients $(\mathrm{n}=47,90.4 \%)$, either isolated or in association with other drugs, followed by antibiotics $(\mathrm{n}=15,28.9 \%)$, paracetamol $(\mathrm{n}=8,15.4 \%)$, and allopurinol $(\mathrm{n}=4,7.7 \%)$.

As shown in Table 1, positive reactions on lesional skin were observed in 21 patients $(40.4 \%)$. Of those, 


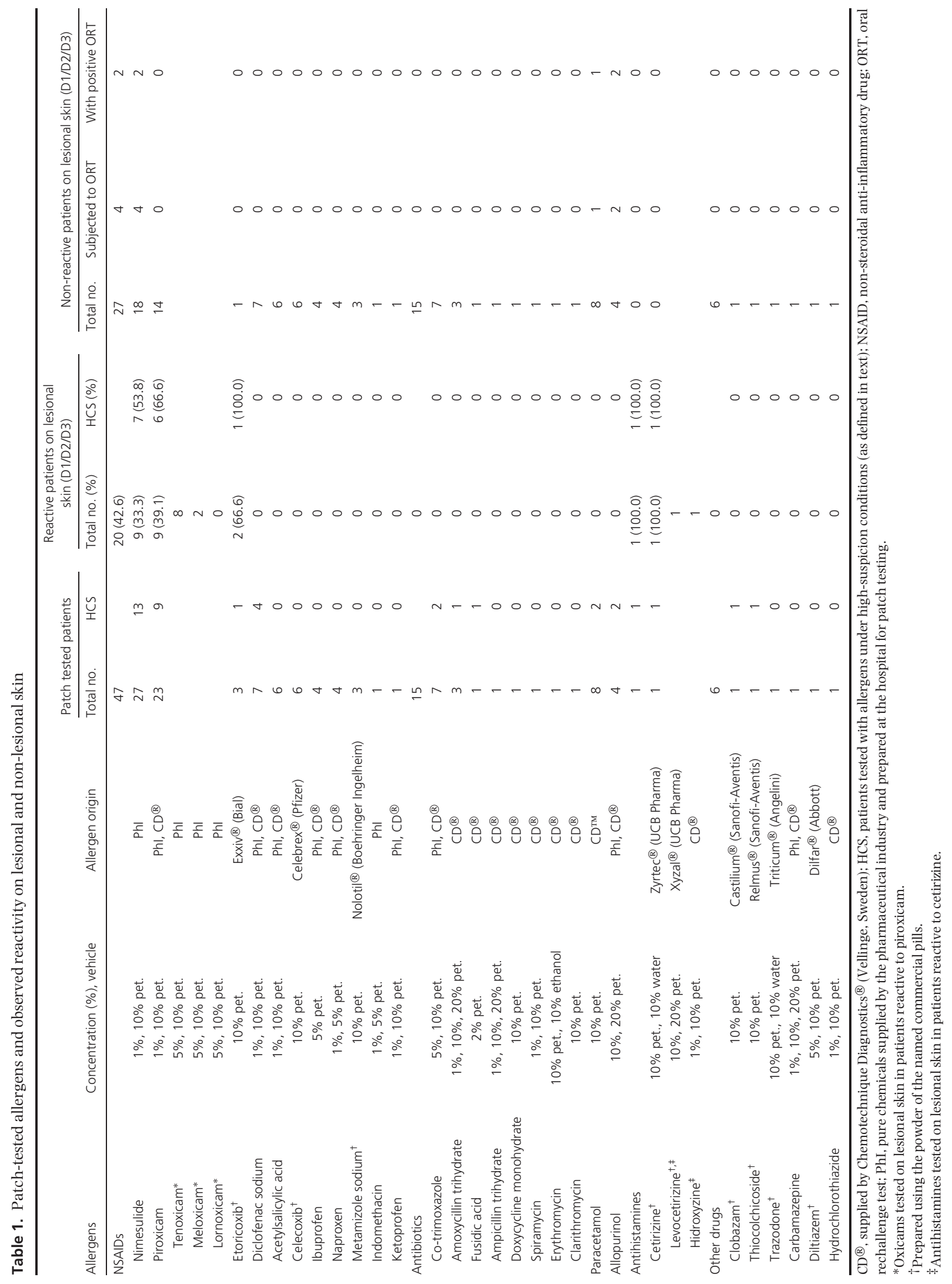


Table 2. Age and sex distribution of patients with fixed drug eruption (FDE) subjected to patch testing

\begin{tabular}{lccccc}
\hline & \multicolumn{2}{c}{ Patch-tested patients } & & \multicolumn{2}{c}{ Female patients } \\
\cline { 2 - 3 } Age group (years) & No. & $\%$ & & No. & $\%$ \\
\hline $0-10$ & 0 & - & & 0 & - \\
$11-20$ & 1 & 1.9 & & 1 & 100.0 \\
$21-30$ & 5 & 9.6 & & 80.0 \\
$31-40$ & 9 & 17.3 & & 7 & 77.8 \\
$41-50$ & 8 & 15.4 & & 4 & 50.0 \\
$51-60$ & 7 & 13.5 & & 57.1 \\
$61-70$ & 14 & 26.9 & & 10 & 71.4 \\
$71-80$ & 8 & 15.4 & & 5 & 62.5 \\
$>81$ & 0 & - & & 0 & - \\
Total & 52 & 100.0 & & 35 & 67.3 \\
\hline
\end{tabular}

20 were reactive to NSAIDs, namely nimesulide $(\mathrm{n}=9)$, piroxicam $(n=9)$, and etoricoxib $(n=2)$. The rate of test positivity in these cases was significantly higher when only patients who were tested under conditions of high clinical suspicion of involvement of a specific drug were considered. Interestingly, of all 9 patients with positive test reactions to piroxicam, 8 were also reactive to tenoxicam and 2 to meloxicam, whereas no reaction was observed to lornoxicam. In addition, none of the other tested NSAIDs induced positive reactions on lesional skin, even when tested in a significant number of patients or under conditions of high clinical suspicion; examples are diclofenac (tested in 7 patients, 4 of them with high clinical suspicion) and acetylsalicylic acid (tested in 6 patients).

The remaining positive test reaction on lesional skin was induced by cetirizine. This patient was tested under conditions of high clinical suspicion of antihistamine involvement, and reacted simultaneously to levocetirizine and hydroxyzine on lesional skin.

Positive patch test reactions on lesional skin were always observed at D1, mostly with erythema and oedema $(\mathrm{n}=12)$, vesicles $(\mathrm{n}=7)$, or even bullae $(\mathrm{n}=2)$, with no significant modification at D2 or D3. Apart from local pruritus on reactivated lesions, no other adverse events were registered. Local inflammation and symptoms were rapidly controlled within a few days by the application of mild topical steroids.

No positive reactions on lesional skin were induced by any of the other drug classes, including antibiotics, not even when allergens were tested in a large number of patients and/or under conditions of high clinical suspicion (Table 1). This was the case for co-trimoxazole (tested in 7 patients, 2 of them with high clinical suspicion), amoxycillin (tested in 3 patients, 1 with high clinical suspicion), paracetamol (tested in 8 patients, 2 with high clinical suspicion), and allopurinol (tested in 4 patients, 2 with high clinical suspicion), among others. Persistent negative results for these drugs were obtained even after performing tape stripping of hyperpigmented lesional patches or increasing the allergen test concentration.

No positive reactions were observed in non-lesional skin tests, except for one 78-year-old female patient who was reactive to piroxicam and tenoxicam, both on lesional and on non-lesional skin.

Oral rechallenge of the suspected drug was undertaken in 7 of the patients with high clinical suspicion of involvement of a specific drug (Table 1), resulting in clinical relapse in most of them (1 patient following re-intake of paracetamol, 2 following re-intake of nimesulide, and 2 following re-intake of allopurinol; Fig. 1); no aggravation was observed in the remaining 2 patients (following reintake of nimesulide). New pruritic lesions were detected in all patients following positive oral rechallenge tests, including mild mucosal lesions in 1 of them; additionally, hyperthermia was observed in 2 of those patients and discrete bullous detachments in 1 . None of these patients required intensive hospital care or surveillance because of the severity of the induced lesions; short-lasting oral steroid therapy was given in 2 of them, and for the remaining 2 patients only topical steroids were prescribed. Total regression of inflammatory lesions was achieved within 1 week in all cases, with new hyperpigmented patches developing.

\section{Discussion}

The present study confirmed NSAIDs as the most frequently suspected drugs in the clinical setting of FDE, and, consequently, the most consistently tested. In our experience, cases in which NSAIDs were not considered were exceptional $(9.6 \%)$, meaning that, in the presence of FDE of unknown origin, NSAID involvement should be always excluded. Systemic antibiotics, paracetamol and allopurinol followed as commonly suspected drugs.

Lesional skin patch test results confirmed the clinical suspicion and allowed the identification of the culprit drug in nearly half of the patients $(40.4 \%)$. Again, following these results, NSAIDs were clearly responsible for $38.5 \%$ of all FDEs. As previously described $(10,11)$, one particular case of FDE was attributed to cetirizine, a well tolerated antihistamine; in this rare case (11), positive reactions to levocetirizine and hydroxyzine were also observed as an expression of cross-reactivity between drugs that have very similar chemical structures. Similarly, simultaneous reactivity to tenoxicam and meloxicam in patients with positive test reactions to piroxicam can also be explained by cross-reactivity phenomena ( 3 , $7,12-15)$. This has also been evaluated in all patients tested for co-trimoxazole, as positive reactions to other 

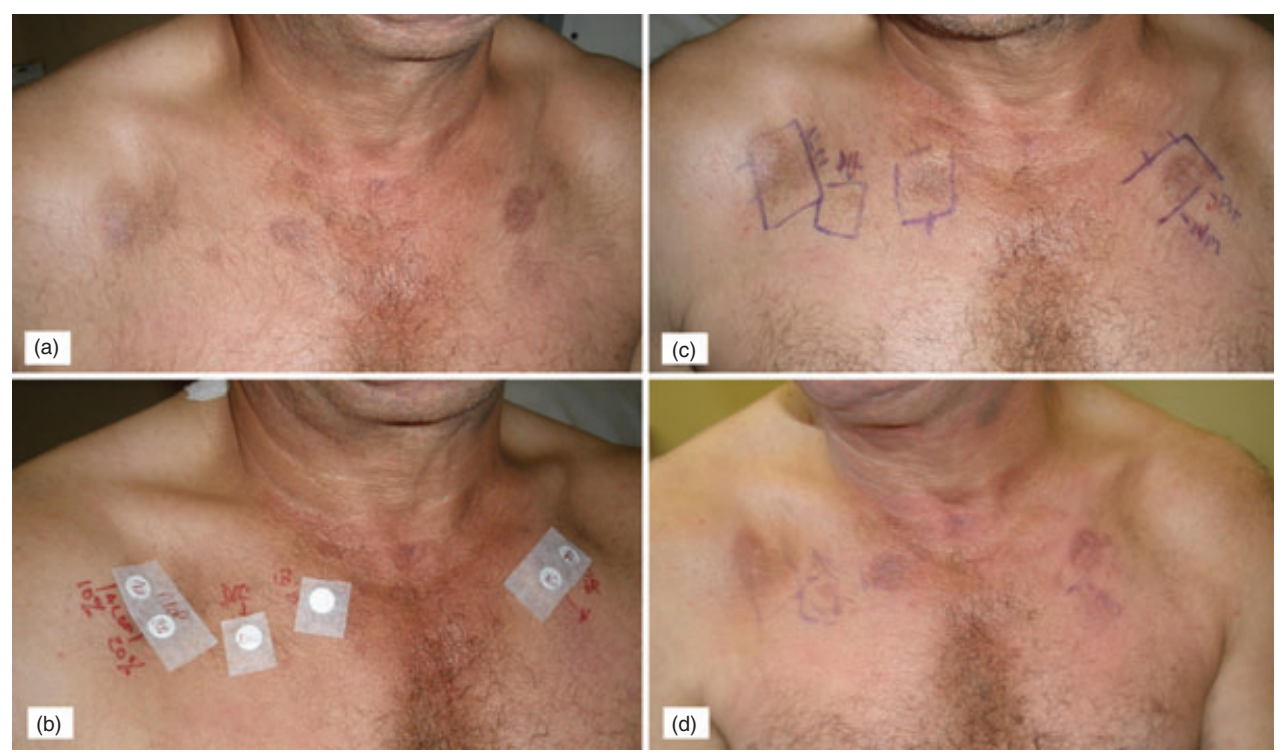

Fig. 1. Patch testing in a fixed drug eruption (FDE) induced by allopurinol. (a) FDE presenting as multiple round residual hyperpigmented macules in the clavicular areas. Non-steroidal anti-inflammatory drugs and allopurinol were the clinically suspected drugs. (b) Patch testing on lesional skin with (from left to right) allopurinol 10-20\% pet., diclofenac sodium 10\% pet., ibuprofen $5 \%$ pet., nimesulide $10 \%$ pet., and piroxicam $10 \%$ pet. (c) Negative patch test results on D1. (d) Positive oral rechallenge test result: recurrence of inflammatory signs on residual lesions and new cervical lesions a few hours after re-exposure to allopurinol $100 \mathrm{mg}$.

sulfonamides were expected $(16,17)$; however, as no positive reactions occurred with this drug, no reactivity was observed with other related allergens. Cross-reactivity is explained by the existence of similar immunogenic chemical structures within different molecules, which are recognized as one by the immune system. Apart from the academic interest, recognition of cross-reactive molecules is undoubtedly important for managing FDE patients $(2,5,13)$, as it might impose additional drug restrictions and allow the suggestion of safe alternatives.

As expected (2), non-lesional skin patch tests gave negative results in nearly all patients. This corroborates the fact that skin-resident memory $\mathrm{CD} 8^{+} \mathrm{T}$ lymphocytes are mostly located in the epidermis of hyperpigmented patches $(2,18)$, explaining the exclusive lesional skin reactivity and the tendency for there to be clinical relapses involving the same areas after oral drug re-exposure (1). The observed exceptional case of positive reactions in both lesional and non-lesional skin is probably attributable to the application of the tests on a non-perceptible normally pigmented post-inflammatory residual lesion, as described in various cases $(1-3,14,19,20)$.

Apart from the expected localized inflammatory signs and pruritus, patch tests did not induce any extension of the FDE lesions, systemic symptoms, or significant adverse events (5). On the other hand, although no significant adverse events were observed after oral drug rechallenge, the evident extension of the disease with associated general symptoms required the use of systemic steroid therapy in some patients, which did not prevent additional residual pigmented patches.

Several previous studies have already demonstrated the importance of NSAIDs as common inducers of FDE (21), often showing variable frequencies in the extent to which they were identified as culprit drugs among other drug classes $(1-4,22)$. Published data from other European countries seem to confirm the role of NSAIDs as the main FDE-inducing drugs, followed by antibiotics and anticonvulsants $(1,22)$, whereas in Korea, muscle relaxants and anticonvulsants seem to be more common inducers of FDE than NSAIDs or co-trimoxazole (8). This variability is probably highly influenced by regional differences in drug prescription $(2,3,8)$. Our results certainly underestimate the real rate of involvement of NSAIDs in FDE, as shown by the occurrence of positive oral NSAID rechallenge test results in patients with negative lesional patch test results with the same allergen. In fact, apart from nimesulide (23), piroxicam (15), and etoricoxib (24), none of the remaining NSAIDs was able to induce positive reactions in patch testing, even when strongly suspected. The same situation was found when antibiotics, paracetamol or allopurinol were considered, even in some of the latter cases where imputability was confirmed by positive oral rechallenge test results. 
In most published studies, NSAIDs seem to be commonly reactive when patch tested on NSAID-related FDE pigmented patches, with positivity rates ranging from $60 \%$ to $100 \%$ for molecules such as phenazone salicylate (22), naproxen (25), and metamizole sodium (25), tested in pet. or in dimethylsulfoxide. Concerning cotrimoxazole, patch test results in FDE seem to be highly variable in most studies, with positivity rates ranging from $60 \%$ to $93 \%$ with dimethylsulfoxide as the vehicle $(22$, 26 ), and from $0 \%$ to $40 \%$ with pet. $(22,26)$. However, as most published studies on patch testing in FDE lack a significant number of patients, and test conditions frequently differ in allergen concentration, vehicles, and patch testing or reading conditions, comparing the available data may be challenging and possibly misleading.

The observed lack of reactivity to some allergens represents a clear limitation to the diagnostic value of patch testing in FDE patients (27). In some cases, it may be attributable to impaired penetration of the specific drug through the stratum corneum (4), preventing activation of latent intraepidermal $\mathrm{CD}^{+} \mathrm{T}$ lymphocytes, which is somehow not compensated for by epidermal thinning methods such as tape stripping or local allergen overload by long-lasting occlusion and increased concentrations. Binding to epidermal proteins, the specific molecular size or lipophilicity may also be important factors determining the potential for percutaneous diffusion (27). Similarly, the vehicle in use might not be appropriate to facilitate the transepidermal migration of the tested allergen $(4,5,28)$, as previously described for co-trimoxazole $(26,29,30)$. Negative results might also be attributable to the fact that the tested molecule is not able to activate the immune response in its primitive form, requiring systemic transformation into its immunologically active metabolite (4). This could be the reason for the absence of reactivity of patch tests with allopurinol, as oxypurinol, its biologically active metabolite, is formed in the liver, and seems to be also the active immunogenic form responsible for other $\mathrm{T}$ cell-mediated cutaneous adverse reactions (31-33). However, in our experience, patch testing with oxypurinol at $5-10 \%$ in different vehicles (pet., ethanol, and acetone) in several cases of cutaneous drug eruptions induced by allopurinol (including FDE) did not change the tendency for there to be negative results, which means that additional different factors may be involved (34). Finally, specific epidermal CD8 ${ }^{+} \mathrm{T}$ lymphocytes might be unable to react to the exposed allergen, as shown by evidence of a refractory period of unknown duration following the onset ofFDE (18). This supports the widely accepted recommendation to perform topical provocation weeks to months after the resolution of acute lesions of FDE, even though it is still unclear for how long the local reactivity potential persists $(5,9)$.

Patch tests constitute a valuable, safe and underexplored tool in the determination of the imputability of suspected drugs in $\operatorname{FDE}(7,27)$. Even though the specificity of the patch test results with commercial drug formulations can be discussed, in FDE this problem is overcome with the negative result 0 non-lesional skin. The lack of sensitivity is, however, a major limitation (27). Precise recommendations of specific patch test conditions for different allergens, in respect of drug concentrations, vehicles, epidermal thinning methods, and duration of occlusion, seems to be crucial for clear interpretation of patch test results, and have not been definitively established for many drugs (5). Non-occlusive patch tests have also been described and associated with interesting results in FDE, questioning the role of allergen occlusion, and suggesting the possibility of short-lasting early positive reactions that might be unnoticed in the classical 24-hr (D1) readings (22). Additionally, criteria must be defined in order to clarify the relevance of mild local reactions such as pruritus or non-infiltrated erythema, which seem not to be predictive of positive drug rechallenge test results $(2,8)$. The use of vehicles other than pet., such as dimethylsulfoxide, or higher concentrations of allergens may play a role in raising the specificity of patch testing for some allergens, but may also induce false-positive reactions, owing to non-specific irritant action on the skin $(3,26)$, necessitating careful result interpretation. Further studies in this area are required, in order to clarify the factors that induce or prevent positive reactions for each considered drug, and to enhance the credibility of this technique in the confirmation of drug imputability.

\section{References}

1 Brahimi N, Routier E, Raison-Peyron N et al. A three-year-analysis of fixed drug eruptions in hospital settings in France. Eur J Dermatol 2010: 20: 461-464.

2 Lee A Y. Fixed drug eruptions. Incidence, recognition, and avoidance. Am J Clin Dermatol 2000: 1: 277-285.
3 Sehgal V N, Srivastava G. Fixed drug eruption (FDE): changing scenario of incriminating drugs. Int J Dermatol 2006: 45: 897-908.

4 Ozkaya E. Fixed drug eruption: state of the art. J Dtsch Dermatol Ges 2008: 6: 181-188.
5 Barbaud A. Drug patch testing in systemic cutaneous drug allergy. Toxicology 2005: 209: 209-216.

6 Lammintausta K, KortekangasSavolainen O. The usefulness of skin tests to prove drug hypersensitivity. $\mathrm{Br}$ J Dermatol 2005: 152: 968-974. 
7 Gonçalo M, Oliveira H S, Fernandes B, Robalo-Cordeiro M, Figueiredo A. Topical provocation in 31 cases of fixed drug eruption. Exogenous Dermatol 2002: 1: $81-86$.

8 Lee A Y. Topical provocation in 31 cases of fixed drug eruption: change of causative drugs in 10 years. Contact Dermatitis 1998: 38: 258-260.

9 Barbaud A, Gonçalo M, Bruynzeel D, Bircher A. Guidelines for performing skin tests with drugs in the investigation of cutaneous adverse drug reactions. Contact Dermatitis 2001: 45: 321-328.

10 Assouère M N, Mazereeuw-Hautier J, Bonafé J L. Cutaneous drug eruption with two antihistaminic drugs of a same chemical family: cetirizine and hydroxyzine. Ann Dermatol Venereol 2002: 129: 1295-1298.

11 Cravo M, Gonçalo M, Figueiredo A. Fixed drug eruption to cetirizine with positive lesional patch tests to the three piperazine derivatives. Int J Dermatol 2007: 46: 760-762.

12 Gastaminza G, Echechipia S, Navarro J A, Fernandéz de Corrés L. Fixed drug eruption to piroxicam. Contact Dermatitis 1993: 28: 43-44.

13 Ordoqui E, De Barrio M, Rodríguez V M, Herrero T, Gil P J, Baeza M L. Cross-sensitivity among oxicams in piroxicam-caused fixed drug eruption: two case reports. Allergy 1995: 50: 741-744.

14 Valsecchi R, Cainelli T. Nonpigmenting fixed drug eruption to piroxicam. J Am Acad Dermatol 1999: 21: 1300.

15 Oliveira H S, Gonçalo M, Reis J P, Figueiredo A. Fixed drug eruption to piroxicam. Positive patch tests with cross-sensitivity to tenoxicam. J Dermatol Treat 1999: 10: 209-212.

16 Strom B L, Schinnar R, Apter A J, Margolis D J, Lautenbach E, Hennessy S,
Biker W B, Pettitt D. Absence of cross-reactivity between sulfonamide antibiotics and sulfonamide nonantibiotics. N Engl J Med 2003: 349: 1628-1635.

17 Zawodniak A, Lochmatter P, Beeler A, Pichler W J. Cross-reactivity in drug hypersensitivity reactions to sulfasalazine and sulfamethoxazole. Int Arch Allergy Immunol 2010: 153: 152-156.

18 Mizukawa Y, Shiohara T. Fixed drug eruption: a prototypic disorder mediated by effector memory T cells. Curr Allergy Asthma Rep 2009: 9: 71-77.

19 Morais P, Baudrier T, Mota A, Cunha A P, Cadinha S, Barros A M, Azevedo F. Nonpigmented fixed drug eruption induced by esomeprazole. Cutan Ocul Toxicol 2010: 29: 217-220.

20 Galindo P A, Borja J, Feo F, Gómez E, Encinas C, García R. Nonpigmented fixed drug eruption caused by paracetamol. J Investig Allergol Clin Immunol 1999: 9: 399-400.

21 Bigby M, Stern R. Cutaneous reactions to nonsteroidal anti-inflammatory drugs. J Am Acad Dermatol 1985: 12: 866-876.

22 Alanko K. Topical provocation of fixed drug eruption. A study of 30 patients. Contact Dermatitis 1994: 31: 25-27.

23 Robalo-Cordeiro M, Gonçalo M, Fernandes B, Oliveira H, Figueiredo A. Positive lesional patch tests in fixed drug eruptions from nimesulide. Contact Dermatitis 2000: 43: 307.

24 Andrade P, Gonçalo M. Fixed drug eruption caused by etoricoxib -2 cases confirmed by patch testing. Contact Dermatitis 2011: 64: 118-120.

25 Ozkaya-Bayazit E. Topical provocation in fixed drug eruption due to metamizol and naproxen. Clin Exp Dermatol 2004: 29: 419-422.
26 Ozkaya-Bayazit E, Bayazit H, Ozarmagan G. Topical provocation in 27 cases of cotrimoxazole-induced fixed drug eruption. Contact Dermatitis 1999: 41: 185-189.

27 Friedmann P S, Ardern-Jones M. Patch testing in drug allergy. Curr Opin Allergy Clin Immunol 2010: 10: 291-196.

28 Hadgraft J, Whitefield M, Rosher P H. Skin penetration of topical formulations of ibuprofen $5 \%$ : an in vitro comparative study. Skin Pharmacol Appl Skin Physiol 2003: 16: 137-142.

29 Oleaga J M, Aguirre A, González M, Diaz-Pérez J L. Topical provocation of fixed drug eruption due to sulphamethoxazole. Contact Dermatitis 1993: 29: 155.

30 Ozkaya-Bayazit E, Güngör H. Trimethoprim-induced fixed drug eruption: positive topical provocation on previously involved and uninvolved skin. Contact Dermatitis 1998: 39: 87-88.

31 Braden G L, Warzynski M J, Golightly M, Ballow M. Cell-mediated immunity in allopurinol-induced hypersensitivity. Clin Immunol Immunopathol 1994: 70: 145-151.

32 Emmerson B T, Hazelton R A, Frazer I H. Some adverse reactions to allopurinol may be mediated by lymphocyte reactivity to oxypurinol. Arthritis Rheum 1988: 31 : 436-440.

33 Hamanaka H, Mizutani H, Nouchi N, Shimizu Y, Shimizu M. Allopurinol hypersensitivity syndrome: hypersensitivity to oxypurinol but not allopurinol. Clin Exp Dermatol 1998: 23 32-34.

34 Vieira R, Gonçalo M, Figueiredo A. Testes epicutâneos ao alopurinol e oxipurinol em doentes com toxidermias ao alopurinol. Trab Soc Port Dermatol Venereol 2004: 62: 247-253. 